\title{
Dynamic Programming for Minimum Steiner Trees
}

\author{
B. Fuchs ${ }^{a}$ W. Kern ${ }^{b}$ D. Mölle ${ }^{c}$ S. Richter ${ }^{c}$ P. Rossmanith ${ }^{c}$ \\ X. Wang ${ }^{b, 1}$

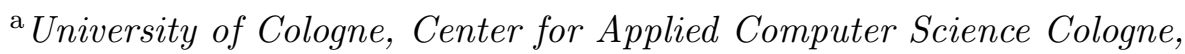 \\ Group AFS, Weyertal 80, 50931 Köln, Germany \\ bfuchs@zpr.uni-koeln.de \\ ${ }^{\mathrm{b}}$ University of Twente, Department of Applied Mathematics, Faculty of EEMCS, \\ P.O.Box 217, 7500 AE Enschede, The Netherlands \\ $\{$ w.kern,xinhuiw\}@math.utwente.nl \\ ${ }^{\mathrm{c}}$ Computer Science Department, RWTH Aachen University, Germany \\ \{moelle,richter,rossmani\}@cs.rwth-aachen.de
}

\begin{abstract}
We present a new dynamic programming algorithm that solves minimum Steiner tree problems with $k$ terminals in time $O^{*}\left(c^{k}\right)$ for any $c>2$. This improves the running time of the previously fastest exponential time algorithms (Dreyfus-Wagner $[2]$ ) of order $O^{*}\left(3^{k}\right)$ and the so-called "full set dynamic programming" algorithm, cf. [3], solving rectilinear instances in time $O^{*}\left(2.38^{k}\right)$.
\end{abstract}

Key words: Steiner tree, exact algorithm, dynamic programming, Dreyfus-Wagner

\section{Introduction}

The Steiner tree problem is one of the most well-known NP-hard problems: Given a graph $G=(V, E)$ of order $n=|V|$, edge costs $c: E \rightarrow \mathbb{R}_{+}$and a set $Y \subseteq V$ of $k=|Y|$ terminals, we are to find a minimum cost tree $T \subseteq E$ connecting all terminals. Note that here and in the following, we identify a subtree of the underlying graph with its edge set $T \subseteq E$. The node set of the

1 Supported by Netherlands Organization for Scientific Research (NWO) grant 613.000.322 (Exact Algorithms). 
tree is denoted by $V(T)$. So an optimal Steiner tree for $Y$ is a tree $T=T(Y)$ that minimizes $c(T)$ subject to $Y \subseteq V(T)$.

The Steiner tree problem has been investigated extensively w.r.t. approximation (cf., e.g., [1]) and computational complexity, both from a theoretical and practical point of view ([3], [7]). The most popular algorithm for computing minimum Steiner trees is the dynamic programming procedure proposed by Dreyfus and Wagner [2] which we shortly present below to make our presentation self-contained.

First note that (since $c \geq 0$ ) every leaf of a minimum Steiner tree must be a terminal. Every interior node is either a terminal or a Steiner node. To describe the Dreyfus-Wagner algorithm, let us adopt the following (rather ambiguous) notation: For $X \subseteq V$ we let $T(X)$ denote both the cost of minimum Steiner tree for $X$ as well as the minimum Steiner tree itself.

The Dreyfus-Wagner algorithm recursively computes $T(X \cup v)$ for all $X \subseteq Y$ and $v \in V$. In the "generic case", the new terminal $v \in V$ is a leaf of the Steiner tree $T=T(X \cup v)$ and $v$ is joined by a min cost path $P_{v w}$ to an interior node $w \in V(T)$ of degree at least 3 . The node $w$ splits $T \backslash P_{v w}$ into two parts, namely $T\left(X^{\prime} \cup w\right)$ and $T\left(X^{\prime \prime} \cup w\right)$ for some nontrivial bipartition $X=X^{\prime} \cup X^{\prime \prime}$. Hence we may write (slightly misusing the notation as announced earlier)

$$
T(X \cup v)=\min \quad P_{v w} \cup T\left(X^{\prime} \cup w\right) \cup T\left(X^{\prime \prime} \cup w\right),
$$

where the minimum is taken over all nontrivial bipartitions $X=X^{\prime} \cup X^{\prime \prime}$ and all $w \in V$.

The above recursion is also valid in the "non-generic cases", when the new terminal $v$ is not a leaf of $T$ (choose $w=v$ ) or when $v$ is joined by $P_{v w}$ to a leaf of $T(X)$, i.e., when $w$ has only degree 2 in $T$ (take $X^{\prime}=\{w\}$ in this case).

Summarizing, the above recursion correctly computes an optimal tree for $Y \subseteq$ $V$. As to the running time, observe that there are less than $n\left(\begin{array}{l}k \\ i\end{array}\right)$ sets of type $X \cup v$ with $|X|=i$ and each such $X$ has less than $2^{i}$ nontrivial bipartitions. Hence we get

$$
n \sum_{i \leq k}\left(\begin{array}{c}
k \\
i
\end{array}\right) 2^{i}=n 3^{k}=O^{*}\left(3^{k}\right)
$$

as an upper bound on the running time.

\section{Improving the exponential time bound}

Let us fix some minimum Steiner tree $T=T(Y)$ for $Y$. Every leaf of $T$ is a terminal. In case $T$ has interior nodes which are terminals, these interior 
terminals split $T$ into components, i.e., maximal subtrees without any interior terminals. The basic idea of our approach is to add (a few) additional terminals so as to ensure that $T$ is split into many "small" components and then recursively reconstruct $T$ from these small components. Here and in the following, the size of a component equals the number of terminals (leaves) of the component.

Lemma 2.1 For $\epsilon>0$, it suffices to add $a \leq 1 / \epsilon$ additional terminals, splitting $T$ into components of size at most $\epsilon k+1$ each.

Proof. We may assume w.l.o.g. that $T$ has no interior terminals. For an interior node $u \in V(T)$, let $k_{u} \leq k-1$ denote the maximum size (including $u$ ) of the components induced by $u$. There exists a node $u$ with $k_{u} \leq k / 2+1$. Hence there also exists a node $u^{*}$ that maximizes $k_{u^{*}}$, subject to $k_{u^{*}} \leq k-k \epsilon$. Observe that $u^{*}$ splits $T$ into one large component of size $k_{u^{*}}$ and one or more components of size (including $u^{*}$ ) at most $\epsilon k+1$ each. By induction, the large component can be split into components of size at most $\frac{\epsilon}{1-\epsilon} k_{u *}+1 \leq \epsilon k+1$ with no more than $\frac{1-\epsilon}{\epsilon}=\frac{1}{\epsilon}-1$ additional terminals.

To describe our algorithm that reconstructs $T$ (or any other optimal Steiner tree for $Y$ ) by successively attaching small components, we adopt the following notation from [3] for terminal sets $X_{1}, X_{2}$ and $X$ :

$$
X:=X_{1} \bowtie X_{2} \quad \Leftrightarrow \quad X=X_{1} \cup X_{2} \text { and }\left|X_{1} \cap X_{2}\right|=1 \text {. }
$$

Assume that $A \subseteq V(T),|A|=\left\lfloor\frac{1}{\epsilon}\right\rfloor$ has been added as a set of additional terminals, splitting $T=T(Y)$ into components of size at most $\epsilon k+1$. Let $\tilde{Y}=Y \cup A$, so that $T=T(\tilde{Y})$. If $X_{1} \subseteq \tilde{Y}$ is the terminal set of a connected union of components, then the subtree of $T$ induced by $X_{1}$ must be a minimum Steiner tree for $X_{1}$ (otherwise $T$ would not be optimal). Hence the following algorithm indeed composes recursively an optimal tree for $Y$ by successively attaching small components, once an appropriate set $A$ of additional terminals is chosen.

\section{Algorithm ASC ("Attach Small Components")}

For each $\tilde{Y}, Y \subseteq \tilde{Y} \subseteq V,|\tilde{Y}|=k+\left\lfloor\frac{1}{\epsilon}\right\rfloor$ do:

1) Compute $T(X)$ for all $X \subseteq \tilde{Y},|X| \leq \epsilon k+1$.

2) For all $X \subseteq \tilde{Y},|X|>\epsilon k+1$, compute $T(X)$ recursively, according to

$$
T(X)=\min \left\{T\left(X_{1}\right) \cup T\left(X_{2}\right)\left|X=X_{1} \bowtie X_{2},\right| X_{2} \mid \leq \epsilon k+1\right\} .
$$

There are $O\left(n^{\frac{1}{\epsilon}}\right)$ choices for $\tilde{Y}$ of size $\tilde{k}=k+\left\lfloor\frac{1}{\epsilon}\right\rfloor$. The time needed for 1$)$ 
(using Dreyfus-Wagner) is negligible for reasonably small $\epsilon>0$. So the total running time is bounded by

$$
n^{\frac{1}{\epsilon}} \sum_{i}\left(\begin{array}{c}
\tilde{k} \\
i
\end{array}\right)\left(\begin{array}{c}
i \\
\epsilon k+1
\end{array}\right) \leq n^{\frac{1}{\epsilon}} \tilde{k} 2^{\tilde{k}}\left(\begin{array}{c}
\tilde{k} / 2 \\
\epsilon \tilde{k}
\end{array}\right) .
$$

This yields our main result.

Theorem 2.1 Algorithm ASC correctly computes a minimum Steiner tree for $Y \subseteq V,|V|=k$ in time $O^{*}\left(c^{k}\right)$ for any $c>2$ by an appropriate choice of $\epsilon>0$.

Proof. By Stirling's Formula, the binomial in (3) can be approximated (up to a polynomial) as

$$
\left(\begin{array}{c}
\tilde{k} / 2 \\
\epsilon \tilde{k}
\end{array}\right) \approx\left[\left(\frac{1}{2 \epsilon}\right)^{\epsilon}\left(\frac{1}{1-2 \epsilon}\right)^{\frac{1}{2}-\epsilon}\right]^{\tilde{k}}<(1+\delta)^{\tilde{k}}
$$

for any prescribed $\delta>0$, provided $\epsilon>0$ is small enough. Hence indeed the running time is $O^{*}\left[(2+2 \delta)^{\tilde{k}}\right]=O^{*}\left[(2+2 \delta)^{k}\right]$.

Remark. The idea of composing the optimal tree from its components has been used by Ganley and Cohoon [4] in the rectilinear case, i.e., when $Y \subseteq \mathbb{R}^{2}$ and $(V, E)$ is the grid graph induced by $Y$ endowed with the Manhattan metric $|x-y|=\left|x_{1}-y_{1}\right|+\left|x_{2}-y_{2}\right|$. The currently fastest algorithms for minimal Steiner tree in the rectilinear case are based on this (de-)composition (cf. [3]). The point is that in the rectilinear case, a lot can be said about the structure of these components. Indeed, it can be assumed w.l.o.g. that each component of the optimal tree consists of a straight line (the Steiner Chain), which starts at a terminal node and has edges (legs) attached to it alternatively from left and right. In addition, the last leg may have an additional edge attached to it, $c f$. Figure 1.

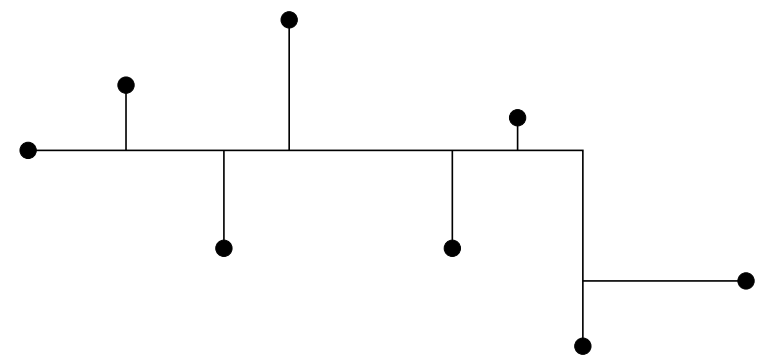

Fig. 1. A component in the rectilinear case

This structure of components in the rectilinear case in known as Hwang topology (cf. [6]). The components in the rectilinear case are called full components and, correspondingly, a subset $X \subseteq Y$ is called a full set if $X$ is the terminal set of a Steiner tree as in Fig. 1, i.e., of a component with Hwang topology. 
Exploiting additional structural properties of full components, Fößmeier and Kaufmann [3] could show that the number of full sets is bounded by $1.38^{k}$. Full sets can be identified easily, so the recursive construction of the optimal tree according to

$$
T(X)=\min \left\{T\left(X_{1}\right) \cup T\left(X_{2}\right) \mid X=X_{1} \bowtie X_{2} \subseteq Y, X_{2} \text { full }\right\}
$$

takes time

$$
\sum_{i}\left(\begin{array}{c}
k \\
i
\end{array}\right) 1.38^{i}=O^{*}\left(2.38^{k}\right)
$$

It remains to be analyzed, whether our idea of splitting the optimal tree can be fruitfully applied to the rectilinear case to yield an algorithm with complexity $O^{*}\left(c^{k}\right)$ without such a prohibitively large polynomial factor of $n^{\frac{1}{\epsilon}}$.

Remark. An analogue of ASC can be designed to solve the directed Steiner tree problem, where the underlying graph $(V, E)$ is a directed graph and we seek for a directed rooted tree (with prescribed root terminal) connecting $Y$. Indeed, it suffices to compute rooted Steiner trees $T_{r}(X)$ (rooted in $r \in X$ ) for all small $X \subseteq \tilde{Y}$ and then attach these successively in the obvious way.

\section{Improving the polynomial factor}

In this section we show how to improve the polynomial factor of $n^{\frac{1}{\epsilon}}$ to roughly $n^{\frac{1}{\sqrt{\epsilon}}}$. The basic idea is as follows. Instead of recursively constructing the optimal tree $T$ by adding components of size $\epsilon k$ in each step, we allow the addition of larger pieces at levels $i \ll k / 2$ and $i \gg k / 2$. Only when $i \approx k / 2$, we proceed by adding small components of size $\epsilon k$ as before.

To work this out in detail, we need the following technical result:

Lemma 2 For sufficiently small $\alpha>0$ and $\epsilon^{\prime}<\alpha^{2}$ we have

$$
\left(\begin{array}{c}
k \\
i
\end{array}\right)\left(\begin{array}{c}
i \\
\epsilon^{\prime} k
\end{array}\right) \leq 2^{k}
$$

for all $i$ such that $|k / 2-i| \geq \alpha k$.

Proof. If suffices to prove the claim for $i=\left(\frac{1}{2}+\alpha\right) k$. By Stirling's Formula, we compute

$$
\left(\begin{array}{c}
k \\
\left(\frac{1}{2}+\alpha\right) k
\end{array}\right)\left(\begin{array}{c}
\left.\left(\frac{1}{2}+\alpha\right) k\right) \\
\epsilon^{\prime} k
\end{array}\right)=\left[\left[\frac{1}{\frac{1}{2}-\alpha}\right]^{\left(\frac{1}{2}-\alpha\right)}\left[\frac{1}{\epsilon^{\prime}}\right]^{\epsilon^{\prime}}\left[\frac{1}{\frac{1}{2}+\alpha-\epsilon^{\prime}}\right]^{\left(\frac{1}{2}+\alpha-\epsilon^{\prime}\right)}\right]^{k}
$$


(up to polynomial factors). Hence, setting $\epsilon^{\prime}=\alpha^{\beta}$ with $\beta>2$, our claim can be restated as

$$
f(\alpha):=\left(\frac{1}{2}-\alpha\right)^{\left(\frac{1}{2}-\alpha\right)}\left(\alpha^{\beta}\right)^{\alpha^{\beta}}\left(\frac{1}{2}+\alpha-\alpha^{\beta}\right)^{\left(\frac{1}{2}+\alpha-\alpha^{\beta}\right)} \geq \frac{1}{2} .
$$

Note that $f(0)=\frac{1}{2}$. Elementary calculus yields

$$
f^{\prime}(\alpha)=f(\alpha)\left[-\ln \left(\frac{1}{2}-\alpha\right)+\beta^{2} \alpha^{\beta-1} \ln \alpha+\left(1-\beta \alpha^{\beta-1}\right) \ln \left(\frac{1}{2}+\alpha-\alpha^{\beta}\right)\right] .
$$

Let $g(\alpha)$ denote the term in brackets. Then $g(0)=0\left(\right.$ as $\left.\lim _{\alpha \rightarrow 0} \alpha^{\beta-1} \ln \alpha=0\right)$ and

$$
\begin{aligned}
g^{\prime}(\alpha)= & \left(\frac{1}{2}-\alpha\right)^{-1}+\beta^{2}(\beta-1) \alpha^{\beta-2} \ln \alpha+\beta^{2} \alpha^{\beta-2}-\beta(\beta-1) \alpha^{\beta-2} \ln \left(\frac{1}{2}-\alpha-\alpha^{\beta}\right) \\
& +\left(1-\beta \alpha^{\beta-1}\right)^{2}\left(\frac{1}{2}-\alpha-\alpha^{\beta}\right) .
\end{aligned}
$$

Now $\beta>2$ implies $\lim _{\alpha \rightarrow 0} \alpha^{\beta-2} \ln \alpha=0$, showing that $g^{\prime}(\alpha) \approx 4>0$ for sufficiently small $\alpha>0$. Hence also $g(\alpha)$ and $f^{\prime}(\alpha)=f(\alpha) g(\alpha)$ are positive for sufficiently small values of $\alpha$. So indeed $f(\alpha) \geq \frac{1}{2}$ for sufficiently small $\alpha>0$.

Let us call - relative to a value of $\alpha$ to be determined below - a level $i$ critical if $|k / 2-i| \leq \alpha k$, and uncritical otherwise. We modify the recursion (2) in ASC such that, as long a $i$ is uncritical, we replace $\epsilon$ by $\epsilon^{\prime}>\epsilon$, whereas for critical $i$, everything remains unchanged. Lemma 2 ensures that our upper bound on the running time of (2) remains unchanged:

$$
\sum_{i \text { uncritical }}\left(\begin{array}{c}
\tilde{k} \\
i
\end{array}\right)\left(\begin{array}{c}
i \\
\epsilon^{\prime} \tilde{k}
\end{array}\right)+\sum_{i \text { critical }}\left(\begin{array}{c}
\tilde{k} \\
i
\end{array}\right)\left(\begin{array}{c}
i \\
\epsilon \tilde{k}
\end{array}\right) \leq \tilde{k} 2^{\tilde{k}}\left(\begin{array}{c}
\tilde{k} / 2 \\
\epsilon \tilde{k}
\end{array}\right) .
$$

Corresponding to the modified recursion, we investigate non-homogeneous subdivisions of the optimal tree $T$ into (many) components of size at most $\epsilon^{\prime} k$ and (a few) components of size at most $\epsilon k$. We first add $\frac{1}{\epsilon^{\prime}}$ additional terminals to ensure component size of at most $\epsilon^{\prime} k$. Now add such components (in some order), one at a time, until the constructed subtree spans $\left(\frac{1}{2}-\alpha\right) \tilde{k}$ terminals.

At this point, we enter the critical phase. We keep adding $\epsilon^{\prime}$-components until the current subtree has $\left(\frac{1}{2}+\alpha\right) \tilde{k}$ terminals. At this point the critical phase stops. The critical subtree, i.e., the subtree of $T$ that we added during the critical phase spans at most $2 \alpha \tilde{k}$ (plus possibly $\epsilon^{\prime} \tilde{k}$ ) terminals. We can subdivide this critical subtree into $\epsilon$-components with at most $(2 \alpha / \epsilon)$ additional terminals. After the critical phase, we complete the optimal tree by adding 
$\epsilon^{\prime}$-components, one at a time. This shows that, in order to make the modified recursion (2) work, it suffices to add

$$
a \leq 2 \alpha / \epsilon+1 / \epsilon^{\prime}
$$

additional terminals.

Now choose $\rho<\frac{1}{2}$ close to $\frac{1}{2}$ and observe that $\alpha:=\epsilon^{\rho}$ and $\epsilon^{\prime}:=\epsilon^{\varsigma}$ with $\varsigma=\frac{1}{2}+\rho$ satisfy the assumption of Lemma 2 (for sufficiently small $\epsilon>0$ ). The number of necessary additional terminals is thus

$$
a \leq 2 \epsilon^{\rho} / \epsilon+\frac{1}{\epsilon^{\varsigma}}
$$

Applying the same trick to $\epsilon^{\prime}$ instead of $\epsilon$, we can further reduce the necessary number of additional terminals to

$$
a \leq 2 \epsilon^{\rho} / \epsilon+2\left(\epsilon^{\rho} / \epsilon\right)^{\varsigma}+\frac{1}{\epsilon^{\varsigma^{2}}} .
$$

Continuing this way, we arrive at

Proposition For every $\rho<1 / 2$, the number of necessary additional terminals can be reduced to $O\left(\epsilon^{\rho-1}\right)$.

Proof. Since $\varsigma=\frac{1}{2}+\rho<1$, there exists a constant $r>0$ such that $\varsigma^{r} \leq 1 / 2$. Hence if we apply our trick $(r-1)$ times, we arrive at

$$
a \leq 2\left[\epsilon^{\rho} / \epsilon+\left(\epsilon^{\rho} / \epsilon\right)^{\varsigma}+\cdots+\left(\epsilon^{\rho} / \epsilon\right)^{\varsigma^{r-1}}\right]+\frac{1}{\epsilon^{\varsigma^{r}}} \leq 2 r \epsilon^{\rho} / \epsilon+\frac{1}{\sqrt{\epsilon}}=O\left(\epsilon^{\rho-1}\right) .
$$

\section{References}

[1] C. Gröpl, S. Hougardy, T. Nierhoff and H. J. Prömel, (2001) Approximation Algorithms for the Steiner Tree Problem in Graphs, in: Steiner Trees in Industries, X. Cheng and D.-Z. Du (eds.), Kluwer.

[2] S. E. Dreyfus and R. A. Wagner, (1972) The Steiner problem in graphs. Networks, $1,195-207$.

[3] U. Fößmeier and M. Kaufmann, (2000) On exact solutions for the rectilinear Steiner tree problem Part 1: Theoretical results. Algorithmica, 26, 68-99.

[4] J. L. Ganley and J. P. Cohoon, (1994) Optimal rectilinear Steiner minimal trees in $O\left(n^{2} 2.62^{n}\right)$ time, in: Proceedings of the Sixth Canadian Conference on Computational Geometry, 308-313. 
[5] M. Garey and D. Johnson, (1977) The Rectilinear Steiner Tree Problem is NPComplete, SIAM Journal on Applied Mathematics 32(4), 826-834.

[6] F. K. Hwang, (1976) On Steiner Minimal Trees with Rectilinear Distance. SIAM Journal on Applied Mathematics, 30(1), 104-114.

[7] D. M. Warme, P. Winter and M. Zachariasen, (2000) Exact Algorithms for Plane Steiner Tree Problems: A Computational Study, in: Advances in Steiner Trees, D.Z. Du, J.M. Smith and J.H. Rubinstein (eds.), Kluwer. 